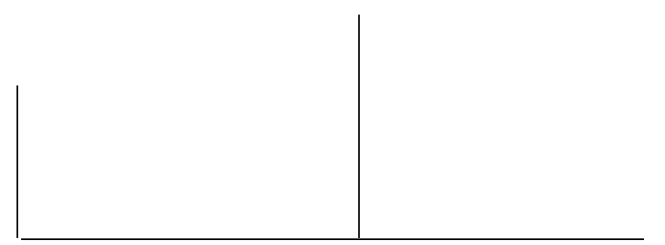

Rev. Latinoam. Psicopat. Fund., VIII, 3, 406-427

\title{
A esquizofrenia ao longo da infância*
}

\author{
Claude Bursztejn
}

\begin{abstract}
As pesquisas sobre os grupos "de alto risco" (filhos de pais esquizofrênicos), assim como os estudos prospectivos na população geral, mostram que os futuros esquizofrênicos apresentam, comparativamente aos sujeitos-controle, atrasos do desenvolvimento psicomotor, déficits cognitivos e algumas particularidades comportamentais. Tais dados parecem confirmar a idéia segundo a qual a esquizofrenia corresponderia a um distúrbio neurodesenvolvimental cuja expressão varia ao longo da vida. As especificidades clínicas e evolutivas dos raros casos de esquizofrenia iniciando-se na infância levam alguns autores a pensar que se trataria de uma entidade específica e cujo pertencimento ao "espectro autista" necessita ainda ser estudado.
\end{abstract}

Palavras-chave: Criança, expressividade psicopatológica, esquizofrenia, espectro autista

* Texto originalmente publicado na revista La Psychiatrie de l'Enfant, Paris: Presses Universitaires de France, v. XLVI, fascículo 2, 2003.

Tradução de Dirce Barroso França e Regina Orth de Aragão. 
A esquizofrenia é um diagnóstico raro no curso da infância: segundo Burd e Kerbeshian (1987), a prevalência seria de 0,2 para 10.000 junto às crianças com menos de 12 anos. Os primeiros episódios psicóticos manifestam-se mais freqüentemente no início da vida adulta ou no fim da adolescência (Häfner et al., 1998). A criança é, contudo, concernida por três questões mais ou menos relacionadas entre elas:

1. Pode-se identificar, no curso da infância, os sinais preditivos anunciadores de uma esquizofrenia que se revelará ao fim da adolescência ou no início da idade adulta?

2. Existe uma esquizofrenia da infância? Quais são as particularidades clínicas e prognósticos?

3. Quais são as relações com a esquizofrenia - do ponto de vista psicopatológico e do ponto de vista evolutivo - das patologias fazendo parte do quadro dos distúrbios do desenvolvimento?

Recordemos que a esquizofrenia é atualmente considerada um diagnóstico sindrômico, proposto com base em um conjunto de critérios.

\section{Resumo dos critérios diagnósticos do DSM-IV}

A) Sintomas característicos: duas (ou mais) das manifestações seguintes são presentes, cada uma durante uma parte significativa de tempo durante o período de um mês:

1. idéias delirantes;

2. alucinações;

3. discurso desorganizado;

4. comportamento gravemente desorganizado ou catatônico;

5. sintomas negativos, por exemplo, rebaixamento afetivo ou perda da vontade.

N.B. - Um só sintoma do Critério A é necessário se as idéias delirantes são bizarras ou se as alucinações consistem em uma voz comentando permanentemente o comportamento ou os pensamentos do sujeito, ou se, nas alucinações, várias vozes conversam entre elas. 


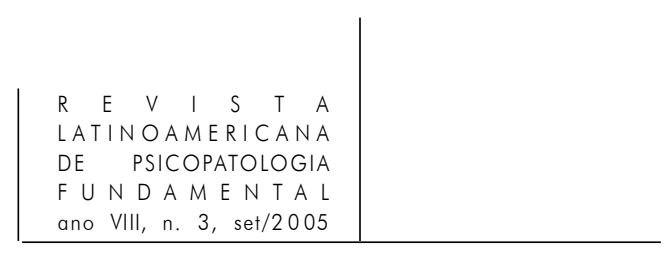

B) Disfuncionamento social / das atividades: durante uma parte significativa do tempo desde o aparecimento da perturbação, um ou vários domínios maiores do funcionamento, tais como o trabalho, as relações interpessoais, ou os cuidados pessoais são nitidamente inferiores ao nível existente antes do aparecimento da perturbação.

C) Duração: os sinais permanentes da perturbação persistem ao menos seis meses.

Exclusão de um problema esquizo-afetivo e de um problema de humor

Exclusão de uma afecção médica geral devido a uma substância

D) Relação com um distúrbio invasivo do desenvolvimento: em caso de antecedente de distúrbio autístico ou de um outro distúrbio invasivo do desenvolvimento, o diagnóstico adicional de esquizofrenia não se faz a não ser que as idéias delirantes ou as alucinações estejam igualmente presentes durante pelo menos um mês.

Existem vários sub-tipos: paranóide, desorganizada, catatônica, indiferenciada e residual.

\section{Esquizofrenia aparecendo na idade adulta}

Os sinais preditivos da esquizofrenia

no curso da infância (sintomas pré-mórbidos)

Muitos estudos retrospectivos interessaram-se pela infância dos esquizofrênicos cujos distúrbios se iniciaram na idade adulta. Ainda que as pesquisas apresentem limites do ponto de vista metodológico, elas mostram, de maneira bastante coerente, que os sujeitos que se tornaram esquizofrênicos apresentaram no curso da infância maior tendência ao recolhimento e mais comportamentos agressivos e anti-sociais do que seus irmãos e irmãs não afetados ou do que os sujeitos-controle. Vários estudos encontraram anomalias do desenvolvimento social, intelectual e neurológico pré-mórbido: um QI rebaixado, retardos no desenvolvimento cognitivo, anomalias sociais foram regularmente observadas particularmente nos meninos; estes sinais são considerados argumentos indiretos em favor da hipótese neuro-desenvolvimental (ver adiante). 
Estes dados foram confirmados por vários estudos prospectivos com sujeitos "com alto risco de esquizofrenia" (quer dizer, tendo um genitor esquizofrênico) que mostraram que os filhos que desenvolveram uma esquizofrenia na idade adulta apresentavam retardos em diferentes domínios do desenvolvimento e uma adaptação social pior do que os sujeitos-controle.

A [pesquisa] New York Infant Study ${ }^{1}$ teve início em 1952 e comparou 12 crianças de mães esquizofrênicas com 12 sujeitos-controle provenientes de famílias de baixo nível socioeconômico. B. Fish (Fish, 1977; Fish et al., 1992;) promotor dessa pesquisa, reagrupou sob o termo "pandismaturação" um conjunto de sinais comportando:

- os retardos transitórios do desenvolvimento motor ou visual, seguidos por uma aceleração e um retorno aos níveis normais;

- um perfil funcional anormal ao exame de desenvolvimento;

- bem como um retardo do crescimento esquelético.

Esta síndrome se observa junto às sete crianças das quais esquizofrenia ou problemas esquizoitípicos foram notados na idade adulta.

O estudo israelense de Marcus et al. (1987) utilizou os mesmos índices de desenvolvimento. Observou-se igualmente a presença desta síndrome indicando dificuldades de amadurecimento em aproximadamente metade dos filhos de pais esquizofrênicos.

Segundo Fish et al. (1992) estas observações sugerem uma "desregulação inata” do desenrolar cronológico e topográfico do desenvolvimento neurológico. Eles propõem diferentes hipóteses sobre as estruturas cerebrais que poderiam ser afetadas, em relação às observações das anomalias histológicas cerebrais reportadas por Kovelman e Scheibel (desorientação das células piramidais do hipocampo). Estas hipóteses se inscrevem no quadro da teoria neurodesenvolvimental de Weinberger.

O "Copenhagen High Risk for Schizophrenia Project”2 (Mednick et al., 1971), lançado em 1962, tem por objeto 207 filhos de mães esquizofrênicas. 31 desenvolveram esquizofrenia; 41 distúrbios de personalidade do tipo esquizoparanóide; e 37 outros problemas psíquicos não psicóticos (avaliação realizada entre 1986 e 1989 com a SADS ${ }^{3}$ e dois outros protocolos de exame estruturado). Olin et al. (1995) analisaram os relatórios de professores recolhidos durante a

1. Em inglês no original. (N. da T.)

2. Em inglês, no original (Projeto Copenhague de Alto Risco para Esquizofrenia). (N. da T.)

3. Schedule for Affective Disorders and Schizophrenia (Escala para Desordens Afetivas e Esquizofrenia). (N. da T.) 


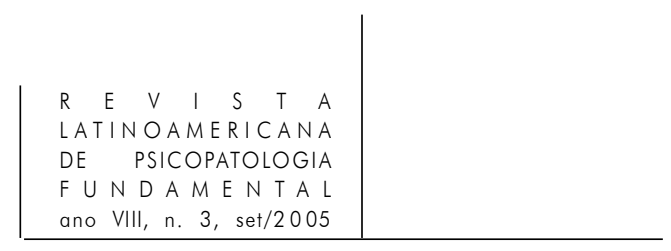

infância junto a 16 sujeitos. Vários itens desses relatórios se revelaram preditivos: os meninos que desenvolveram posteriormente esquizofrenia, eram descritos como crianças solitárias, rejeitadas pelos outros, tendo comportamentos inadaptados e tendo apresentado problemas de disciplina. As meninas que desenvolveram uma esquizofrenia eram qualificadas como nervosas e passivas. Os professores, em uma grande proporção, haviam julgado essas crianças como de "risco de distúrbios psicóticos” no futuro.

O “New York High Risk Project - 1971”4 (Erlenmeyer-Kimling, 1997) tem por objeto 79 filhos de pais esquizofrênicos, 57 filhos de pacientes afetados por distúrbios maiores de humor e 133 sujeitos-controle. Além de recolher dados comportamentais, uma bateria de provas cognitivas (escala Wechsler, testes de atenção, de memória a curto prazo, escala de desenvolvimento neuromotor) foi administrada às crianças com 9 anos de idade. Os déficits de memória verbal, de atenção e de motricidade global foram observados nos sujeitos que apresentaram uma psicose esquizofrênica na idade adulta; o estudo estatístico mostrou que a combinação desses três déficits tinha valor preditivo.

Estes dados são convergentes com aqueles dos estudos de grupos da população geral, realizados na Grã-Bretanha (Cannon et al., 1999): mostram igualmente que os futuros esquizofrênicos se diferenciam dos sujeitos não afetados por retardos do desenvolvimento psicomotor, déficits cognitivos e particularidades comportamentais.

Segundo Cannon et al. (1999), existe mesmo uma concordância entre a sintomatologia pré-mórbida e o tipo de esquizofrenia apresentada posteriormente pelo sujeito. Em seu estudo - que repousa sobre as observações dos professores - os esquizofrênicos adultos com uma predominância de sintomas negativos, tiveram significativamente mais comportamentos pré-mórbidos "do tipo negativo" (isto é, tendência à solidão, a ser rejeitado, a ser tímido e passivo na escola) do que os pacientes nos quais os sintomas positivos eram predominantes. Inversamente, os esquizofrênicos com sintomatologia positiva predominante tinham apresentado significativamente mais comportamentos pré-mórbidos do "tipo positivo" (isto é, mais excitáveis, irritáveis ou agressivos) do que os esquizofrênicos nos quais os sintomas negativos predominavam.

Para esses autores, os resultados sugerem a existência de duas síndromes esquizofrênicas distintas. É necessário notar, entretanto, que este estudo não contempla senão sete esquizofrênicos com sintomas negativos predominantes e oito com sintomas positivos predominantes, o que limita o valor desta pesquisa.

4. Em inglês, no original (Projeto New York de Alto Risco). (N. da T.) 


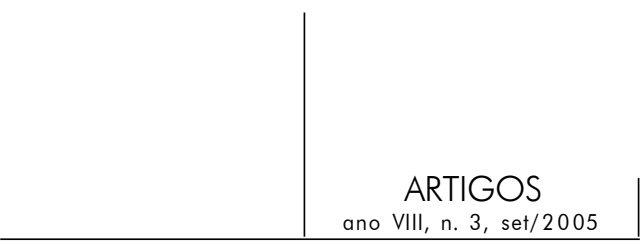

Baum e Walker (1995) retomaram esta questão interrogando os pais de 29 esquizofrênicos. Os questionários tiveram por objeto o comportamento durante a infância até os 15 anos de todos os seus filhos e a sintomatologia daqueles que dentre eles tornaram-se esquizofrênicos. Os sintomas eram avaliados a partir de versões adaptadas aos pais da Escala de Avaliação dos Sintomas Positivos (SAPS) e da Escala de Avaliação de Sintomas Negativos (SANS). Os resultados indicam que o déficit psicomotor e a desorganização cognitiva nos pacientes adultos são positivamente associados aos comportamentos de retraimento e inversamente associados aos traços ansiodepressivos no curso da infância. Para os autores, seus resultados confirmam a noção de uma relação diferencial específica dos sintomas adultos com os comportamentos precursores no curso da infância, que corresponderia a um modelo trifatorial da esquizofrenia (déficit psicomotor / desorganização cognitiva/distorção da realidade).

Outros estudos indicam igualmente que o estado pré-mórbido é preditivo do prognóstico da esquizofrenia na idade adulta. O estudo de Bailer et al. (1996) tendo por objeto 163 primeiras admissões de pacientes esquizofrênicos seguidos durante cinco anos (idade média 29,7 anos; 17-59 anos). O estado pré-mórbido era avaliado pelo PAS (Premorbid Adjustment Scale) que estuda a educação recebida, o trabalho e as mudanças profissionais, a freqüência e os resultados escolares, assim como a aquisição de autonomia social. Os resultados sugerem a existência de dois fenótipos esquizofrênicos diferentes:

- o primeiro tipo caracterizado por um nível baixo de adaptação social prémórbida, um início insidioso e uma predominância de sintomas negativos;

- um segundo, caracterizado por uma boa adaptação social pré-mórbida, um início agudo e a ausência de sintomas negativos duradouros.

Na conclusão desses estudos, a maior parte dos autores adere à concepção de Weinberger (1987) que concebe a esquizofrenia como um distúrbio neurodesenvolvimental cuja expressão varia no curso da vida. Segundo este modelo, uma patologia cerebral sobrevém no curso dos primeiros anos de vida. O processo causal permaneceria latente durante um longo período antes do diagnóstico (o mais freqüente no fim da adolescência e início da vida adulta); a aparição dos sintomas característicos estaria ligada à maturação normal das áreas cerebrais afetadas pela patologia desenvolvimental precoce. Segundo esta hipótese, as lesões cerebrais precoces fixadas interagiriam com certos acontecimentos que sobreviriam mais tarde no curso da maturação. A manifestação de anomalias cognitivas, comportamentais ou neurológicas no curso da infância, dos sujeitos que se tornaram esquizofrênicos, refletiria "as anomalias sutis do desenvolvimento cerebral precoce". 


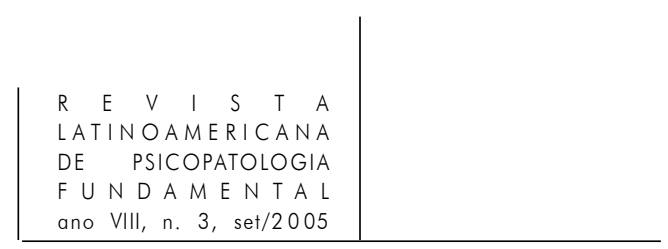

Fase prodrômica da esquizofrenia na adolescência e idade adulta

Numerosos estudos (para uma revisão, ver Glashan e Johannessen, 1996) se interessam pelos sintomas sobrevindos no curso do ano que precede a aparição dos primeiros sintomas psicóticos (geralmente, no fim da adolescência ou início da vida adulta). Para Glashan e Johannessen (1996) esta fase prodrômica deve ser levada em consideração na "duração total da doença". O interesse por esse período foi estimulado pela comprovação, nos estudos retrospectivos e prospectivos, de uma influência negativa da "duração da doença antes do tratamento" sobre o prognóstico e taxa de recidiva (Larsen et al., 1998; Glashan e Johannessen, 1996). Este efeito desfavorável da "duração da doença antes do tratamento" parece confirmado pelos estudos com base no acaso de Crow et al. (1986) e Wyatt et al. (1997); a mesma tendência - ainda que não estatisticamente significativa - foi encontrada no estudo longitudinal de dois anos de Craig et al. (2000). É necessário notar, contudo, que em uma pesquisa de Verdoux et al. (no prelo) o efeito da "duração da doença antes do tratamento" sobre a evolução aparece com menos evidência quando se tem em conta o nível de funcionamento pré-mórbido e a intensidade dos sinais negativos adultos.

No estudo de Jackson et al. (1995) que teve por objeto os 313 primeiros episódios psicóticos, o sintoma mais freqüentemente observado durante a fase prodrômica é o retraimento social; os outros sintomas esquizofrênicos (comportamento bizarro, falta de cuidados, afetos rebaixados ou inapropriados, etc.) são menos regularmente encontrados e podem se observar junto a pacientes para os quais outros diagnósticos foram feitos. Mas nos 13 primeiros episódios esquizofrênicos Yung e McGorry (1996) indicam a freqüência de sintomas ainda menos específicos: distúrbios do sono, ansiedade, irritabilidade, humor depressivo, idéias suicidas, distúrbios obsessivo-compulsivos, rebaixamento da atenção e da capacidade de concentração, apatia, "anhedonismo". ${ }^{5}$

Paralelamente, programas de intervenção precoce foram propostos. O mais freqüentemente citado é o de Falloon (1992) que implantou entre 1984 e 1989, no condado de Buckingham, um dispositivo de detecção pelos médicos generalistas. Aqueles tinham a possibilidade de desencadear uma intervenção rápida (algumas horas após a chamada!) da equipe de saúde mental. Essas intervenções comportavam cuidados em domicílio (visando principalmente o controle do estresse) e incluíam o ambiente familiar. Neurolépticos de baixa dose eram prescritos em função dos sintomas-alvo, mas não de modo sistemático. Segundo a es-

5. Anhédonie, em francês - neologismo criado em 1896 por Thédule Ribot para designar a insensibilidade ao prazer. (N. da T.) 


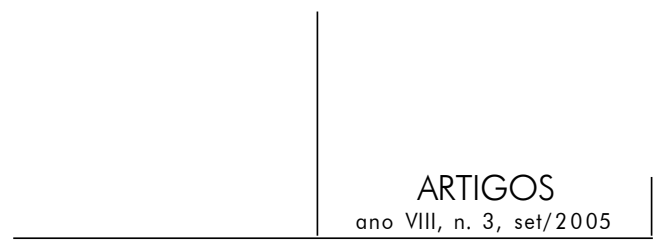

timativa do autor, a incidência anual de esquizofrenia, durante o estudo, foi dez vezes menor: 0,75 para 100.000 , contra 7,5 para 100.000 durante os anos precedentes na mesma região. Não se pode, contudo, excluir as flutuações devidas ao acaso. Glashan e Johannessen (1996) concluem que os dados atuais "sugerem, mas não demonstram” que as intervenções precoces melhoram a evolução da esquizofrenia; eles sublinham a parte preponderante de intervenções psicossociais no estudo de Falloon.

O interesse destas intervenções precoces permanece em discussão. Os argumentos a favor e contra foram sistematicamente examinados por Yung e McGorry (1997), que evocam em particular os problemas éticos que elas suscitam e o risco de efeitos iatrogênicos (estigmatização social, efeitos negativos dos neurolépticos etc.) que elas comportam, especialmente para os inevitáveis “falsopositivos” e, mais particularmente, na adolescência. Lembram que a sintomatologia no curso da fase prodrômica é bastante variada e comporta, come se viu, muitos sintomas não específicos, suscetíveis de serem observados em outros contextos patológicos (distúrbios depressivos ou ansiosos). Eles esperam minimizar o risco do “falso positivo" tomando em consideração, além dos sintomas, a presença de fatores de risco (antecedentes familiares de distúrbios psicóticos, retraimento social, deterioração da performance escolar). Discutem assim os aspectos econômicos, na medida em que a detecção e as intervenções precoces são procedimentos custosos; lembram, enfim, que para aqueles que defendem o modelo neuro-desenvolvimental, o prognóstico depende essencialmente de fatores neurobiológicos, sobre os quais a precocidade de intervenção não tem efeito.

Todas as questões se colocam com uma particular acuidade na adolescência, sobretudo os problemas diagnósticos em relação aos quais se conhece a dificuldade nessa idade. Com efeito, uma grande parte dos sintomas prodrômicos assinalados por Yung e McGorry (notadamente os sintomas negativos, a tendência ao isolamento social) podem se observar transitoriamente junto aos adolescentes, e seria prejudicial eles serem excessivamente - como pode ter sido o caso no passado - considerados sinais anunciadores de uma entrada na esquizofrenia. Além disso, é necessário lembrar a diversidade de evoluções a longo prazo dos episódios psicóticos agudos podendo levar, inclusive, aos distúrbios de humor (Johnson-Sabine et al. 1993).

\section{A esquizofrenia da criança}

O estudo da esquizofrenia começando na infância é considerado pela maior parte dos autores atuais uma aproximação privilegiada na perspectiva da teoria 


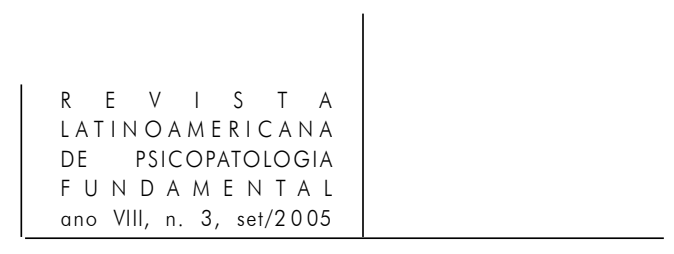

neuro-desenvolvimental da esquizofrenia. Os casos de início muito precoces poderiam representar um grupo mais homogêneo e mais severamente atingido que estaria submetido mais fortemente à ação de um agente etiológico hipotético.

A noção de que os distúrbios psicóticos análogos àqueles observados em adultos possam sobrevir em jovens crianças é muito antiga, pois desde $1906 \mathrm{~S}$. Sanctis havia relatado observações de "demências muito precoces" ocorrendo na infância. Em seguida, após a reformulação por Bleuler da concepção psicopatológica da esquizofrenia, este diagnóstico foi aplicado a um grande número de casos apresentando, em graus diversos, comportamentos relacionais perturbados ou inabituais, distúrbios de modulação das emoções e dos afetos, uma angústia importante e distúrbios do pensamento.

Se Kanner em 1943 - e paralelamente Asperger, mas de modo menos preciso - individualizaram dentre essas crianças um grupo particular correspondendo ao que se designa atualmente sob a expressão "distúrbios autísticos”, as relações desse grupo com a esquizofrenia não foram de imediato esclarecidas mesmo pelo próprio Kanner.

Werry et al. lembram que de 1960 a 1980, na literatura anglo-saxônica, a expressão childhood schizophrenia ${ }^{6}$ foi utilizada nas classificações oficiais para designar todas as psicoses da infância, o que torna difícil a interpretação de certos estudos antigos. É necessário, além disso, lembrar que o termo "esquizofrenia" permaneceu durante muito tempo o único diagnóstico de distúrbios psicóticos proposto para a criança na classificação americana e somente a partir do CID-9 (1970) o termo “autismo infantil” fez sua aparição na classificação internacional.

É sobretudo a partir dos trabalhos de Kolvin (1971) e de Rutter (1972) que se impôs na literatura uma separação nítida entre o autismo e a esquizofrenia. Esta posição prevaleceu tanto no DSM-III e IV quanto no CID-10 que impõem, ademais, a aplicação à criança dos mesmos critérios para a esquizofrenia que os impostos para os adultos. Doravante, é, portanto, a partir desses critérios que é considerada a questão da esquizofrenia no curso da infância.

É necessário lembrar também que as classificações atuais deixam de lado um grande número de crianças cujos problemas, ainda que severos, não correspondem a nenhuma das categorias identificadas do capítulo dos "distúrbios invasivos do desenvolvimento". Estes casos aos quais, à falta de melhor, certas publicações de língua inglesa fazem referência sob o termo PDD NOS (Pervasive Developmental Disorders Non Otherwise Specified ou Distúrbios Invasivos do Desenvolvimento não Especificados) correspondem em grande parte às desarmonias psicóticas da Classificação francesa, que constituem uma parte 


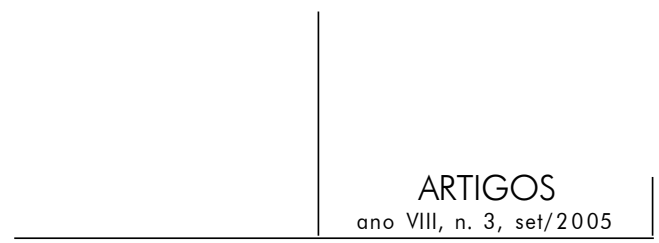

importante dos consultados e das crianças tratadas pelas equipes de psiquiatria infantil. Os estudos recentes abordam este aspecto importante da patologia sob o termo Multiple Developmental Disorders ou Multiple Impaired Disorders; ${ }^{7}$ outros autores, notadamente Gillberg, propõem que sejam incluídos no quadro da síndrome de Asperger (com critérios ampliados). A situação desses casos, em relação à esquizofrenia, mas também quanto aos distúrbios invasivos do desenvolvimento, é atualmente objeto de discussões e de pesquisas.

\section{Os sintomas esquizofrênicos na criança}

A maior parte dos trabalhos recentes considera, portanto, a esquizofrenia sobrevindo no curso da infância e adolescência como uma simples variante clínica. Entretanto, a raridade - ou a dificuldade de comprovação - de certos sintomaschave da esquizofrenia na criança foi sublinhada desde longa data. Os estudos já antigos (Kolvin, 1971; Kydd e Werry, 1982; Eggers, 1982) mostraram que os sintomas negativos (isolamento social, rebaixamento da performance escolar; afetos rebaixados ou inapropriados, pobreza do discurso e perda da iniciativa) predominam sobre os sintomas positivos, notadamente o delírio. Os sintomas negativos são os primeiros a aparecer (fase prodrômica).

Por outro lado, o prognóstico é, em geral, considerado mais desfavorável do que para a esquizofrenia adulta, com 20 a 30\% somente de remissões (Eggers, 1982; Westermeyer e Harrow, 1986; Asarnow et al., 1996) Kydd e Werry (1982) referem um bom prognóstico junto a $40 \%$ dos sujeitos de seu estudos, contudo a evolução a longo prazo mostrou que um grande número de casos incluídos neste estudo se revelaram, com efeito, distúrbios de humor do tipo bipolar. Como mostraram Werry et al. (1991), a partir de um estudo com 59 crianças e adolescentes psicóticos (idade entre 16-17; média de 13,9) mais da metade dos sujeitos apresentavam um distúrbio bipolar tendo recebido um diagnóstico de esquizofrenia quando de sua primeira admissão ou consulta. Estes dados foram confirmados pelos trabalhos recentes (Volkmar et al.,1988; Russel et al., 1989; Werry et al., 1991; Green et al., 1992; Volkmar, 1996).

Mesmo dentro do quadro de critérios do DSM-III e IV ou do CID-10 (Green et al., 1992), a maior parte das pesquisas sobre a esquizofrenia no curso da infância demonstra diferenças notáveis com os grupos de pacientes adultos:

- predominância masculina;

- maior proporção de inícios insidiosos;

- sintomatologia menos diferenciada (menos delírios);

7. Idem. 


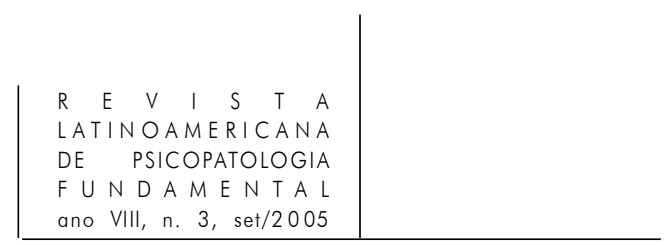

- predomínio de anomalias neuro-desenvolvimentais nos antecedentes;

- maior proporção de antecedentes familiares de esquizofrenia;

- predomínio de personalidades mal adaptadas antes do aparecimento do distúrbio;

- maior resistência ao tratamento antipsicótico;

- prognóstico mais desfavorável.

Estas particularidades sugerem uma especificidade, ao menos do ponto de vista clínico, da esquizofrenia iniciada na infância. É a posição marcada pela Classificação francesa dos distúrbios mentais da infância e do adolescente que diferencia a esquizofrenia da criança da esquizofrenia do adolescente (e do adulto) (Misès e Quemada, 1993).

\section{Anomalias neuropsicológicas}

Hendren et al. (1995) compararam 12 crianças com idades entre 8 e 12 anos, apresentando distúrbios de personalidade esquizoitípica e/ou sintomas fazendo parte do quadro de uma esquizofrenia de início precoce (Escala Kiddie-SADS) e 13 crianças igualadas pela idade, sexo e nível socioeconômico, recrutadas nas escolas ou serviços de pediatria. Os exames comportavam uma bateria de testes neuropsicológicos mostrando resultados inferiores àqueles do grupo-controle sobre os testes de exploração das funções frontais, as capacidades verbais e nãoverbais, assim como a memória verbal e não-verbal. No grupo estudado, observam igualmente no IRM ${ }^{8}$ diferenças de volume das amígdalas, das regiões temporais e dos corpos calosos, comparativamente ao grupo-controle. Para os autores, estes dados vão no sentido do modelo neuro-desenvolvimental da esquizofrenia, que postula que os acontecimentos de origem ambiental ou programados geneticamente perturbam, no curso da vida intra-uterina, o estabelecimento de certos elementos fundamentais da estrutura e da função cerebral.

\section{Dados epidemiológicos}

A prevalência da esquizofrenia iniciando-se na infância é pouco conhecida: ela parece ser, em todo caso, bem inferior àquela dos distúrbios autísticos. Werry (1992) diferencia, por outro lado, as esquizofrenias pré-pubertárias - por vezes designadas sob a expressão "esquizofrenia de início muito precoce” (Very Early Onset Schizophrenia) as mais raras e também as mais severas - e as esquizofrenias pós-pubertárias cujas características se aproximam mais da esquizofrenia adulta.

8. Imagem por ressonância magnética. (N. da T.) 


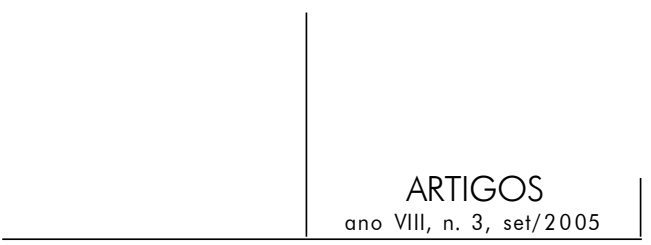

Esquizofrenias e distúrbios invasivos do desenvolvimento

O NIMH ${ }^{9}$ recentemente relançou o estudo da esquizofrenia pré-pubertária. Uma enquete foi divulgada por anúncio em jornais médicos. Dos 260 relatórios recebidos, 186 foram rejeitados em razão da ausência de sintomas psicóticos comprovados (36\%), um início após a idade de 12 anos (10\%) ou outros fatores de exclusão (retardo mental, doenças somáticas ou distúrbios afetivos maiores). Os critérios diagnósticos utilizados eram aqueles do DSM-III-R, salvo para o diagnóstico de síndrome de Asperger, para a qual os critérios do CID-10 foram aplicados.

McKenna et al. (1994) estudaram os 71 casos restantes (6 a 18 anos), cujos primeiros sintomas psicóticos apareceram antes da idade de 12 anos (mas nem sempre antes da puberdade). Um grupo de 19 pacientes, na maioria do sexo masculino, respondiam plenamente aos critérios da esquizofrenia: apresentavam uma sintomatologia próxima daquela dos pacientes adultos com afetos rebaixados e inapropriados, alucinações e idéias delirantes, assim como distúrbios do pensamento. O início havia sido, mais freqüentemente, insidioso ou sub-agudo. Os pródromos comportavam geralmente uma deterioração das performances escolares, um retraimento social, comportamentos desorganizados. A história prémórbida dessas crianças esquizofrênicas ia de uma aparência de normalidade a algumas anomalias do desenvolvimento (distúrbios de linguagem, ansiedade, hipersensibilidade).

Neste estudo são referidos quatro casos de síndrome de Asperger que apresentavam “sintomas psicóticos”. Em um caso as alucinações visuais estavam presentes. Uma outra criança apresentava comportamentos repetitivos pouco habituais, e verbalizações idiossincráticas que foram considerados, pelo médico que a encaminhou, sintomas psicóticos.

Entre os outros relatórios, pareceu necessário ao grupo de pesquisa isolar um grupo ao qual o diagnóstico “Distúrbios multidimensionais” (Multidimensionally Impaired Disorder - Kumra et al., 1998) foi provisoriamente atribuído. Essas 21 crianças apresentaram, antes da idade de 7 anos, retardos ou distúrbios de desenvolvimento e sintomas psicóticos que não correspondiam aos critérios do DSM-III da esquizofrenia. Elas evocavam experiências alucinatórias em resposta a questões do Kiddie-SADS. Contudo, um exame mais aprofundado revelou que essas experiências não eram verdadeiras alucinações ou não eram invasivas. Eles também apresentavam, freqüentemente, uma atividade fantasmática excessiva e julgada inapropriada para sua idade (sobre a base da seção 


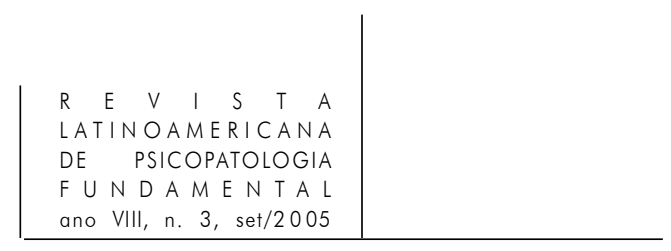

“Delírio” do Kiddie-SADS-E). Além disso, essas crianças apresentavam uma instabilidade de humor com crises de cólera quase cotidianas (avaliadas pelas respostas aos itens concernentes à depressão do Kiddie-SADS-P e do Kiddie-SADS-C, assim como da seção "distúrbios de conduta" do DICA-P). A despeito de suas tentativas de estabelecer relações com os pares, essas crianças tinham graves dificuldades de socialização, mas sem os distúrbios massivos de relações recíprocas, característicos dos "distúrbios invasivos do desenvolvimento".

Todos apresentavam, além disso, um ou dois sintomas que faziam parte dos distúrbios de conduta, dos distúrbios de personalidades-limite ou do tipo esquizóide; mas eles não atendiam aos critérios de nenhuma dessas categorias. 85\% dentre eles respondiam igualmente aos critérios do "distúrbio deficitário de atenção com hiperatividade".

Enfim, eles apresentavam déficits neuropsicológicos de moderados a severos (notadamente viso-espacial ou da vertente receptiva da linguagem). Contrariamente às crianças esquizofrênicas, os neurolépticos tiveram poucos efeitos positivos.

As similitudes deste grupo com as crianças borderlines descritas por outros autores, ou ainda com aquelas descritas sob o termo "distúrbios complexos e múltiplos do desenvolvimento” (MDD) são sublinhadas pelos autores.

Eles concluem com sua pesquisa que o diagnóstico "esquizofrenia iniciada na infância” permanece uma categoria válida e útil, mas que corre o risco de ser utilizada em excesso, em razão da ausência de categoria que agrupe as crianças que apresentam distúrbios psicóticos intermitentes e déficits de desenvolvimento.

\section{Manifestações prodômicas e pré-mórbidas da esquizofrenia da infância}

Há poucos estudos descrevendo os pródromos ou a fase pré-psicótica das esquizofrenias iniciadas na infância.

Kolvin (1971), junto a 33 crianças esquizofrênicas cuja doença havia se desenvolvido antes da idade de 15 anos, havia encontrado anomalias do comportamento entre $87 \%$ dos casos e retardos das principais etapas do desenvolvimento entre $49 \%$ dos casos.

Kydd e Werry (1982) descreveram 15 crianças nas quais a esquizofrenia se desenvolveu antes dos 16 anos. Nos quatro casos em que o início fora insidioso, os sintomas depressivos haviam precedido de um mês a dois anos a eclosão da esquizofrenia.

Asarnow e Ben-Meir (1987) avaliaram retrospectivamente vários parâmetros de adaptação social de crianças que apresentaram uma esquizofrenia (10), 


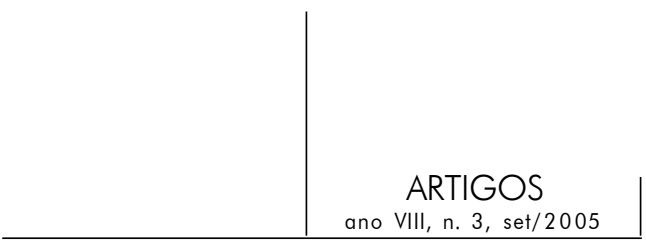

distúrbios esquizoitípicos (11), uma depressão maior (26) e distúrbios distímicos (9). Observaram uma diferença significativa entre o grupo que apresentava distúrbios "pertencentes ao espectro da esquizofrenia e aqueles que apresentavam distúrbios tímicos, com base nos seguintes parâmetros: sociabilidade e tendência ao retraimento, a relação com os pares, os resultados escolares, adaptação ao meio escolar; o início mais insidioso junto às crianças que apresentam distúrbios pertencentes ao espectro da esquizofrenia, comparado ao das crianças que tiveram distúrbios depressivos.

Watkins et al. (1988) em um estudo realizado com 18 crianças esquizofrênicas (critérios do DSM-III-R) cujos distúrbios haviam se iniciado antes dos 11 anos assinalaram igualmente distúrbios sociais, distúrbios de atenção, assim como uma restrição dos afetos e uma hipersensibilidade à crítica antes do aparecimento dos sintomas esquizofrênicos. Graves déficits de linguagem e um retardo do desenvolvimento motor se apresentaram em $72 \%$ dos casos; por outro lado, 39\% das crianças da amostra haviam correspondido aos critérios de autismo infantil antes do início da esquizofrenia. Russel (1989), em outro estudo proveniente do mesmo centro, encontrou que $26 \%$ das 35 crianças que haviam recebido o diagnóstico de esquizofrenia haviam apresentado sintomas de distúrbios invasivos do desenvolvimento, ainda que nenhuma tivesse preenchido completamente os critérios.

Podemos aproximar esta observação daquela de Cantor et al. (1982) que, junto a 30 crianças e adolescentes que apresentavam um diagnóstico de esquizofrenia, encontrou sete casos que previamente haviam recebido um diagnóstico de autismo.

No estudo japonês de Iida, os casos onde existiam os sintomas obsessivos no curso da fase prodrômica são caracterizados por uma forte predominância masculina, uma acentuada incidência de anomalias perinatais e de distúrbios neurológicos e uma duração da fase prodrômica mais longa e correspondente a um subtipo particular. É necessário notar, entretanto, que este estudo realizado com 39 pacientes concerne principalmente à esquizofrenia na adolescência: idade média de 14,6 (desvio de 1,17); nenhum sujeito com menos de 11 anos no momento do início dos distúrbios.

O estudo de Alaghband-Rad et al. (1995) que se inscreve no âmbito da pesquisa realizada pela NIMH, refere-se a 23 crianças de 10 a 19 anos (idade média 14 anos; 15 garotos e 8 garotas) que apresentaram os sintomas psicóticos antes dos 13 anos e interessa-se mais especificamente pela severidade dos distúrbios pré-mórbidos. Retardos de desenvolvimento foram observados entre um quarto à metade dos sujeitos. Nove dos 21 casos apresentaram notadamente um retardo de linguagem moderado (idade média de aparecimento da primeira frase: 26,5 meses). Nove (36\%) haviam apresentado ao menos alguns sinais do tipo autísti- 


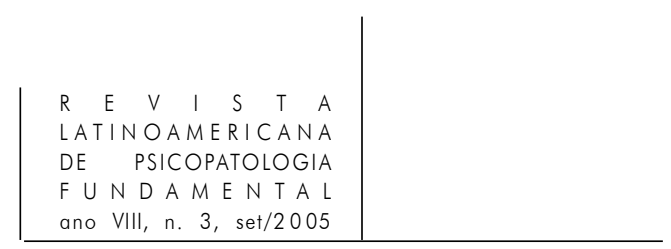

co; em três (13\%) os critérios completos para o autismo foram preenchidos. Sete (30\%) foram considerados apresentando uma hiperatividade com distúrbios de atenção.

Por ordem de freqüência, os sintomas prodrômicos foram os seguintes:

- retraimento, 65\%;

- diminuição dos resultados escolares, 65\%;

- comportamento idiossincrático, 59\%;

- desleixo, 29\%;

- afetos rebaixados ou inapropriados, 71\%;

- linguagem vaga ou pobre, $43 \%$;

- ausência marcada de iniciativa, 53\%;

- pensamentos estranhos ou mágicos, 35\%;

- experiência sensorial estranha, 35\%.

Junto a 15 pacientes (62\%), o início da psicose fora insidioso, enquanto em oito casos (35\%) o início fora subagudo.

Em comparação com os trabalhos sobre a infância das esquizofrenias que tiveram início na vida adulta, os dados deste estudo indicam uma evolução prémórbida mais severa nas formas iniciadas na infância.

Em resumo, o conjunto dos dados de que dispomos atualmente confirma a existência de particularidades clínicas e evolutivas da esquizofrenia iniciada na infância. Todos os autores sublinham a importância dos retardos de desenvolvimento, notadamente no domínio da linguagem, sem que sintomas específicos permitam identificar as crianças com risco de evolução esquizofrênica.

Os dados mais recentes levam a rediscutir as relações nosográficas e psicopatológicas da esquizofrenia com os distúrbios invasivos do desenvolvimento (especialmente a síndrome de Asperger); eles confirmam o interesse da individualização de um grupo específico (MID, MDD Multiple Developmental Disorders e Multiple Impaired Disorders, ou desarmonia psicótica, segundo os autores).

\section{Conclusão}

As pesquisas sobre os grupos de "alto risco" (crianças de pais esquizofrênicos), assim como as pesquisas prospectivas na população geral, mostram que os futuros esquizofrênicos apresentam retardos do desenvolvimento psicomotor, déficits cognitivos e idiossincrasias comportamentais, comparativamente aos sujeitos-controle. Esses dados parecem confirmar o modelo de Weinberger segundo o qual a esquizofrenia seria um distúrbio neuro-desenvolvimental cuja 
expressão varia no curso da vida. As particularidades clínicas e evolutivas desses casos raros de esquizofrenia iniciadas na infância (predominância de sintomas negativos, início geralmente insidioso, prognóstico mais desfavorável) levam certos autores a considerá-la uma patologia específica e não uma simples variante clínica. O estudo dos casos de esquizofrenia com início muito precoce recoloca a questão das formas de passagem com certos distúrbios pertencentes ao “espectro autístico” (especialmente a síndrome de Asperger).

Primavera de 2002

\section{Referências}

Alaghband-Rad, J.; McKenna, K.; Gordon, C. T.; Albus, K. E.; Hamburger, S. D.; Rumsey, J. M.; Frazier, J. A.; Lenane, M. C.; Rapoport, J.-L. Childhood-onset schizophrenia: The severity of premorbid course. Journal of the American Academy of Child \& Adolescent Psychiatry, n. 34, p. 1273-83, 1995.

Asarnow, J. R. \& Ben-Meir, S. Children with schizophrenia spectrum and depressive disorders: A comparative study of premorbid adjustment, onset pattern and severity of impairment. Journal of Child Psychology and Psychiatry, n. 29, p. 477-88, 1988.

Asarnow, J. R.; Tompson, M. C.; Goldstein, M. J. Childhood onset schizophrenia: A follow-up study. Schizophrenia Bulletin, n. 20, p. 599-617, 1994.

Bailer, J.; Brauer, W.; Rey, E. R. Premorbid adjustment as predictor of outcome in schizophrenia: Results of a prospective study. Acta Psychiatr. Scand., n. 93, p. 368-77, 1996.

Baum, K. M. \& Walker, E. F. Childhood behavioral precursors of adult symptom dimensions in schizophrenia. Schizophen. Res., n. 16, 111-20, 1995.

Cannon, M.; Jones, P.; Huttunen, M. O.; Tanskanen, A.; Huttunen, T.; Rabe-Hesketh, S.; Murray, R. M. School performance in Finnish children and later development of schizophrenia: A population-based longitudinal study. Archives of General Psychiatry, v. 56, n. 5, p. 457-63, May 1999.

Caplan, R. Thought disorder in childhood. Journal of the American Academy of Child \& Adolescent Psychiatry, n. 33, p. 605-15, 1994.

Craig, T. J.; Bromet, E. J.; Fennig, S.; Tanenberg-Karant, M.; Lavelle, J.; Galambos, $\mathrm{N}$. Is there an association between duration of untreated psychosis and 24-month clinical outcome in a first-admission series? Am. J. Psychiatry, n. 157, p. 60-6, 2000.

Crow, T. J.; McMillan, J. F.; Johnson, A. L.; Johnstone, E. C. A randomised controled trial of prophylactic neuroleptic treatment. Brit. J. Psychiatry, n. 148, p. 120-27, 1986.

Done, D. J.; Crow, T. F.; Johnstone, E. C.; SAcker, A. Childhood antecedents of schizo- 


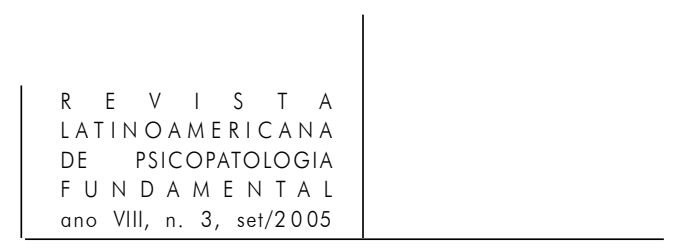

phrenia and affective illness: Social adjustment at ages 7 and 11. Brit. Med. J., n. 309, p. 699-703, 1994.

EGGERs, C. Course and prognossis of childhood schizophrenia. Journal of Autism \& Childhood Schizophrenia, n. 8, p. 21-35, 1978.

Erlenmeyer-Kimling, L.; Adamo, U. H.; Rock, D.; Roberts, S. A.; Bassett, A. S.; Squires-Wheeler, E.; Cornblatt, B.; Endicott, J.; Pape, S.; Gottesman, I. I. The New York high risk project: Prevalenceand comorbidity of axis I disorders in offspring of schizophrenic parents at 25 year of follow-up. Archives of General Psychiatry, n. 54, p. 1096-102, 1997.

Erlenmeyer-Kimling, L.; Rock, D.; Roberts, S. A.; Janal, M.; Kestenbaum, C.; Cornblatt, B.; Adamo, U. H.; GotTesman, I. I. Attention, memory, and motor skills as childhood predictors of schizophrenia-related psychoses: The New York high risk project. Am. J. Psychiatry, n. 157, p. 1416-22, 2000.

Erlenmeyer-Kimling, L.; Squires-Wheeler, E.; Adamo, U. H.; Bassett, A. S.; Curnblatt, B.; Kestenbaum, C.; Rock, D.; Roberts, S. A.; Gottesman, I. I. The New York high risk project: Psychoses and cluster. A personality disorders in offspring of schizophrenic parents at 23 years of follow-up. Archives of General Psychiatry, n. 52, p. 857-65, 1995.

Fennig, S.; Susser, E. S.; Pilowsky, D. J.; Fennig, S.; Bromet, E. J. Childhood hallucinations preceding the first psychotic episode. J. Nerv. Ment. Dis., n. 185, p. 115-7, 1997.

Fish, B. Neurobiologic antecedents of schizophrenia in children: Evidence for an inherited, congenital neurointegrative defect. Archives of General Psychiatry, n. 34, p. 1297-1313, 1977.

Fish, B.; Marcus, J.; Hans, S. L.; Auerbach, J. G.; Perdue, S. Infants at risk for schizophrenia: Sequelae of a genetic neurointegrative defect. A review and replication analysis of pandysmaturation in the Jerusalem infant development study. Archives of General Psychiatry, n. 49, p. 221-35, 1992.

Foerster, A.; Lewis, S. W.; Owen, M. J.; Murray, R. M. Low birth weight and a family history of schizophrenia predict poor premorbid functioning in psychosis. Schipophr. Res., n. 5, p. 13-20, 1991.

Franke, P.; Maier, W.; Hardt, J.; Hain, C. Cognitive functining and anhedonia in subjects at risk for schizophrenia. Schizoph. Res., n. 10, p. 77-84, 1993.

Glashan, T. H. \& JohanNESSEN, J. O. Early detection and intervention with schizophrenia: rationale. Schizophrenia Bulletin, n. 22, p. 201-22, 1996.

Green, W.; Padron-Gayol, M.; Hardesty, A.; Bassiri, M. Schizophrenia with childhood onset: A phenomenological study of 38 cases. Journal of the American Academy of Child \& Adolescent Psychiatry, n. 31, p. 968-76, 1992.

Hafner, H.; Hambrecht, M.; Loffler, W.; Mullk-Jorgense, P.; Riecher-Rossler, A. Is 


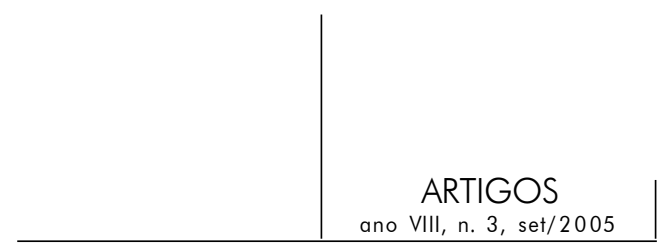

schizophrenia a disorder of all ages? A comparison of first episodes and early course across the life-cycle. Psychol. Med., n. 28, p. 351-65, 1998.

Hebebrand, J.; Henninghausen, K.; Nau, S.; Himmelmann, G. W.; Schulz, E.; Schafer, H.; Remschmidt, H. Low body weight in male children and adolescent with schizoid personality disorder or asperger's disorder. Acta Psychiatr. Scand., n. 96, p. 64-7, 1997.

Hendren, R. L.; Hodde-Vargas, J.; Yeo, R. A.; Vargas, L. A.; Brooks, W. M.; Ford, C. Neuropsychophysiological study of children at risk fur schizophrenia: A preliminary report. Journal of the American Academy of Child \& Adolescent Psychiatry, n. 34, p. 1284-91, 1995.

Iida, J.; Inasaka, H.; Hirao, F.; Hashino, K.; Matsumura, K.; Tahara, K.; Aoyama, F.; SAKIYAMA, S.; Tsujimoto, H.; KaWABATA, Y.; IKaWA, G. Clinical features of childhood-onset schizophrenia with obsessive-compulsive symptoms during the prodromal phase. Psychiatr. Clin. Neurosci., n. 49, p. 201-7, 1995.

Jackson, H. J.; McGorry, P. D.; Dakis, J.; Harrigan, S.; Henry, L.; Mihalopoulos, C. The inter-rater and test-retest reliabilities of prodromal symptom in first-episode psychosis. Australian \& New Zealand Journal of Psychiatry, n. 30, p. 498-504, 1996.

Jackson, H. J.; McGorry, P. D.; Dudgeon, P. Prodromol symptoms of schizophrenia in first-episode psychosis: prevalence and specificity. Compr. Psychiatry, n. 36, p. 24150, 1995.

Johnson-Sabine, E. C.; Mann, A. H.; Jacoby, R. J.; Wood, K. H.; Péron-Magnan, P.; Olie, J.-P.; Deniker, P. Bouffée délirante: An examination of its current status. Psychological Medicine, n. 13, p. 77l-8, 1993.

Jones, P.; Rodgers, B.; Murray, R.; Marmot, M. Child dcvelopmental risk factors for adult schizophrenia in the British 1946 birth cohort. Lancet, n. 344, p. 1398-402, 1994.

Keshavan, M. S.; Schooler, N. R.; Sweeney, J. A.; HaAs, G. L.; Pettegrew, J. W. Research and treatment strategies in first-people psychoses. The Pittsburgh experience. British Journal of Psychiatry - Supplement, n. 172, p. 60-5, 1998.

Kolvin, I. Studies in childhood psychoses. I - Diagnostic criteria and classification. Brit. J. Psychiatry, n. 118, p. 381-4, 1971.

Kovelman, J. A. \& Scheibel, A. B. A neurohistological correlate of schizophrenia. Biological Psychiatry, n. 19, p. 1607-21, 1984.

Kumra, S.; Jacobsen, L. K.; Lenane, M.; Zahn, T. P.; Wiggs, E.; Alaghband Ruavad; Castellanos Fxavier; Frazier, Jean A.; McKenna, K.; Gordon, C. T.; Smith, A.; HamBURGER, Susan; RAPOPORT, J.-L. "Multidimensionally impaired disorder”: Is it a variant of very early-onset schizophrenia? Journal of the American Academy of Child \& Adolescent Psychiatry, n. 37, p. 91-9, 1998.

KydD, R. R. \& Werry, J. S. Schizophrenia in children under 16 years. Journal of Au- 


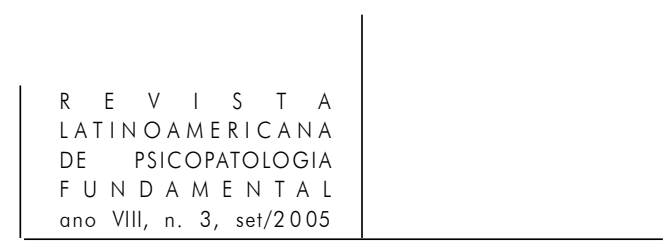

tism and Developmental Disorders, n. 12, p. 343-57, 1982.

Larsen, T. K.; Johannessen, J. O.; OpJordsmoen, S. First-episode schizophrenia with long duration of untreated psychosis. Pathways to care. British Journal of Psychiatry Supplement, n. 172, p. 45-52, 1998.

Larsen, T. K.; McGlashan, T. H.; Johannessen, J. O.; Vibe-Hansen, L. First-episode schizophrenia. II - Premorbid patterns by gender. Schizophrenia Bulletin, n. 22, p. 25769, 1996.

Lieb, K.; Denz, E.; Hess, R.; Schuttler, R.; Kornhuber, H. H.; Schreiber, H. Preattentive information processing as measured by backward masking and texton detection tasks in adolescents at high genetic risk for schizophrenia. Schizophen Res., n. 21, p. 17182, 1996.

Marcus, J.; Auerbach, J. G.; Wilkinson, L.; Burack, C. M. Infants at risk for schizophrenia: The Jerusalem infant development study. Archives of General Psychiatry, n. 34, p. 703-13, 1981.

Marcus, J.; Hans, S. L.; Nagler, S.; Auerbach, J. G.; Mirsky, A. F.; Aubrey, A. Review of the NIMH Israeli Kibbutz-city study and the Jerusalem infant development study. Schizophrenia Bulletin, n. 13, p. 425-38, 1987.

Maziade, M.; Gingras, N.; Rodrigue, C.; Bouchard, S.; Cardinal, A.; Gauthier, B.; Tremblay, G.; Cote, S.; Fournier, C.; Boutin, P.; Hamel, M.; Roy, M. A.; Martinez, M.; Merette, C. Long-term stability of diagnosis and symptom dimensions in a systematic sample of patients with onset of schizophrenia in childhood and early adolescence. I - Nosology, sex and age of onset. Brit. J. Psychiatry, n. 169, p. 361-70, 1996.

McGlashan, T. H. \& Johannessen, J. O. Early detection and intervention with schizophrenia: Rationale [132 refs]. Schizophrenia Bulletin, n. 22, p. 201-22, 1996.

McKenna, K.; Gordon, C. T.; Lenane, M. C.; Kaysen, D. et al. Looking for childhoodonset schizophrenia: The first 71 cases screened. Journal of the American Academy of Chid \& Adolescent Psychiatry, n. 33, p. 636-44, 1994.

Mednick, S. A.; Mura, M.; Schulsinger, F.; Mednick, B. Perinatal conditons and infant development in children with schizophrenic parents. Soc. Biology, n. 18, p. S103-S113, 1971.

Misès, R. \& QuemadA, N. Classification française des troubles mentaux de l'enfant et de l'adolescent. 3. éd. Vanves: CTNERHI, 1993.

Montero, I.; Ruiz Perez, I.; Gomez-Beneyto, M. Social adjustment in schizophrenia: Factors predictive of short-term social adjustment in a sample of schizophrenic patients. Acta Psychiatr. Scand., n. 97, p. 116-21, 1998.

Olin, S. C. S. \& Mednick, S. A. Risk factors of psychosis: Identifying vulnerable populations premorbidly (Early detection and intervention in Schizophrenia). Schizophre- 


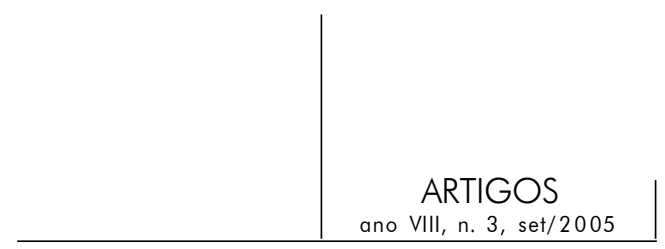

nia Bulletin, n. 22, p. 223-40, 1996.

Russell, A. T.; Bott, L.; Sammons, C. The phenomenology of schizophrenia occuring in childhood. Journal of the American Academy of Child \& Adolescent Psychiatry, n. 28, p. 399-407, 1989.

RutTER, M. Childhood schizophrenia reconsidered. Journal of Autism Developmental Disorders, n. 2, p. 315-37, 1972.

Schmidt, M.; Blanz, B.; Dippe, A.; Koppe, T.; Lay, B. Course of patients diagnosed as having schizophrenia during first episode occurring under age 18 years. European Archives of Psychiatry \& Clinical Neuroscience, n. 245, p. 93-100, 1995.

Su Chin Serene Olin; John, R. S.; Mednick, S. A. Assessing the predictive value of teacher reports in a high risk sample for schizophreniaoe: a ROC analysis. Schizophen. Res., n. 16, p. 53-66, 1995.

Szymanski, S.; Lieberman, J. A.; Alvir, J. M.; Mayerhoff, D.; Loebel, A.; Geisler, S.; Chakos, M.; Koreen, A.; Jody, D.; Kane, J. et al. Gender differences in onset of illness, treatment response, course, and biologic indexes in first-episode schizophrenic patients. Am. J. Psychiatry, n. 152, p. 698- 703, 1995.

Thomsen, H. Borderline conditions in childhood. A register-base follow-up study over a 22-year period. Psychopathology, n. 29, p. 357-62, 1996.

Tompson, M. C.; Asarnow, J. R.; Hamilton, E. B.; Newell, L. E.; Goldstein, M. J. Children with schizophrenia-spectrum disorders: Thought disorder and communication problems in a family interactional context. Journal of Child Psychology \& Psychiatry \& Allied Disciplines, n. 38, p. 421-29, 1997.

Towbin, K.; Dykens, E.; Pearson, G.; Cohen, D. Conceptualizing "borderline syndrome of childhood" and "childhood schizophrenia" as a developmental disorder. Journal of the American Academy of Child \& Adolescent Psychiatry, n. 32, p. 775-82, 1993.

Van der Gaag, R. J.; Buitelaar, J.; Van den Ban, E.; Bezemer, M.; Nito, L.; Van ENGELAND, H. A controlled multivariate chart review of multiple complex developmental disorder. Journal of the American Academy of Child \& Adolescent Psychiatry, n. 34, p. 1096-106, 1995.

Verdoux, H.; Liraud, F.; Bergey, C.; Assens, F.; Abalan, F.; Os, J. van. Is the association between duration of untreated psychosis and outcome confounded? A two-year follow-up study of first-admitted patients. Schizophrenia Bulletin, n. 49, p. 231-41, 2001.

Verdoux, H.; Liraud, F.; Gonzales, B.; Fournet, O.; Pauillac, P.; Assens, F.; Abalan, F.; Beaussier, J.-P.; Gaussares C.; Etchegaray, B.; Bourgeois, M. Prognostic à court terme lors de la première hospitalisation pour trouble psychotique. Encéphale, n. 25, p. 213-20, 1999. 


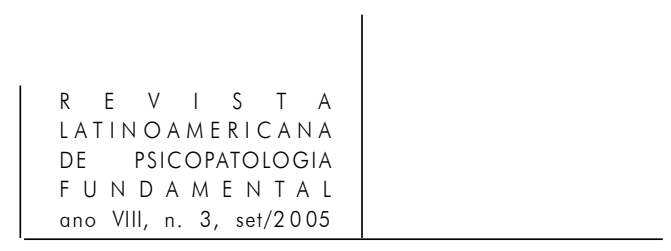

VolKmar, F. Childhood and adolescent psychosis: A review of the past 10 years. Journal of the American Academy of Child \& Adolescent Psychiatry, n. 35, p. 843-51, 1996.

WatKins, J.; Asarnow, R.; TAnguay, P. Symptom development schizophrenia. Journal of Child Psychology \& Psychiatry \& Allied Disciplines, n. 29, p. 865-78, 1988.

WEINBERGER, D. R. The pathogenesis of schizophrenia: A neurodevelopmental theory. In: NasRallah, H.A.; WeInBERGER, D.R. (eds.). The handbok of schizophrenia, I: The neurology of schizophrenia. New York: Elsevier Science Publishers, 1986.

WERRY, J. S. Child and adolescent (early onset) schizophrenia: A review in light of DSMIII-R. Journal of Autism and Developmenlal Disorders, n. 22, p. 601-25, 1992.

Werry, J. S.; McClellan, J. M.; Chard, L. Chidhood and adolescent schizophrenic, bipolar, and schizoaffective disorders: A clinical and outcome study. Journal of the American Academy of Child \& Adolescent Psichiatry, n. 30, p. 457-65, 1991.

Westermeyer, J. F. \& Harrow, M. Predicting outcome in schizophrenics and nonschizophrenics of both sexes: The Zigler-Phillips social competence scale. Journal of Abnormal Psychology, n. 95, p. 406-9, 1986.

Wyatt, R. J.; GreEn, M. F.; Tuma, A. H. Long-term morbidity associated with delayed treatment of first admission schizophrenic patients: A re-analysis of the Camarillo State Hospital data. Psychol. Med., n. 27, p. 261-8, 1997.

YANG, P. C.; LiU, C. Y.; ChianG, S. Q.; CHEN, J. Y.; LiN, T. S. Comparison of adult manifestations of schizophrenia with onset before and after 15 years of age. Acta Psychiatr. Scand., n. 91, p. 209-12, 1995.

Yung, A. R.; McGorry, P. D.; McFarlane, C. A.; Jackson, H. J.; Patton, G. C.; RakKar, A. Monitoring and care of young people at incipient risk of psychosis. Schizophrenia Bulletin, n. 22, p. 283-303, 1996.

YunG, A. R. \& McGorRY, P. D. Is pre-psychotic intervention realistic in schizophrenia and related disorders? [see comments] [21 refs]. Australian \& New Zealand Journal of Psychiatry, n. 31, p. 799-805, 1997.

Yung, A. R. \& McGorRY, P. D. The initial prodrome in psychosis: descriptive and qualitative aspects. Australian \& New Zealand Journal of Psychiatry, n. 30, p. 587-99, 1996.

\section{Resumos}

Los trabajos de investigación sobre grupos "de alto riesgo" (hijos de padres esquizofrénicos), así como los estudios prospectivos en la populación general, muestran que los futuros esquizofrénicos presentan, comparativamente a los sujetos-control, 


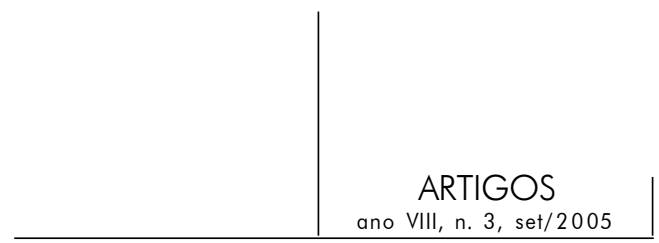

atrasos del desarrollo motor, déficit cognitivos y algunas particularidades de comportamiento. Esos datos parecen confirmar la idea de que la esquizofrenia correspondería a un disturbio de neuro-desarrollo cuya expresión varia a lo largo de la vida. Las especificidades clínicas y evolutivas de los raros casos de esquizofrenia que se inician en la infancia llevan algunos autores a pensar que se trataría de una entidad específica y cuya pertenencia al "espectro autista” necesita ser todavía estudiado.

Palabras claves: Niños, expresividad psicopatológica, esquizofrenia, espectro autista

Les recherches sur les groupes "à haut risque" (enfants de parents schizophrènes), ainsi que des enquêtes prospectives en population générale montrent que les futurs schizophrènes présentent, comparativement aux témoins, des retards du développement psychomoteur, des déficits cognitifs et certaines particularités comportementales. Ces données semblent confirmer l'idée selon laquelle la schizophrénie correspondrait à un trouble neuro-développemental dont l'expression varie au cours de la vie. Les spécificités cliniques et évolutives des rares cas de schizophrénie débutant dans l'enfance amènent certains auteurs à penser qu'il s'agirait d'une entité spécifique et dont l'appartenance au "spectre autistique" demande encore à être envisagée.

Mots clés: Enfant, expressivité psychopathologique, schizophrénie, spectre autistique

The researches on "high risk" groups (children of schizophrenic parents), just like the prospective studies in the general population, show that the future schizophrenics present, comparatively to the "control-subjects", delays on their psychomotor development, cognitive deficits and some behavioral particularities. Such data seem to confirm the idea from which the schizophrenia would correspond to a neurodevelopmental disturb whose expression varies along one's life. The clinical and developmental intricacies of the rear cases of schizophrenia that begin during childhood make some authors think that it could be related to a particular entity and whose belonging to the "autistic spectrum" still needs to be researched.

Key words: Child, psychological expressivity, schizophrenia, autistic spectrum 